\title{
WEB SEMÂNTICA E ONTOLOGIAS: UM ESTUDO SOBRE CONSTRUÇÃO DE AXIOMAS E USO DE INFERÊNCIAS
}

\author{
WEB SEMÁNTICA Y ONTOLOGÍAS: UN ESTUDIO \\ SOBRE LA CONSTRUCCIÓN DE AXIOMAS Y EL USO DE \\ INFERENCIAS
}

\author{
José Eduardo Santarem Segundo* \\ Caio Saraiva Coneglian**
}

\begin{abstract}
RESUMO:
Introdução: A Web Semântica apresenta tecnologias e conceitos que perpassam por questões como descrição, representação e consultas dos dados. Neste cenário, as ontologias contextualizam as informações, permitindo geração de inferências. A linguagem OWL possui características que facilitam a inserção de lógica nos dados, sendo um elemento essencial para a Web Semântica e o Linked Data. Contudo, questiona-se: como essas inferências podem ser visualizadas e utilizadas na Web Semântica.

Objetivo: Analisar os tipos de inferência no contexto das tecnologias da Web Semântica, e consequentemente ao Linked Data.

Metodologia: Consistiu-se em duas partes, sendo a primeira um levantamento bibliográfico para discussão do tema, e na segunda um estudo exploratório baseado em provas de conceitos e testes de uso de inferências.

Resultados: Identificamos e apresentamos quatro formas de realização de inferências, fazendo testes que comprovem sua aplicação.

Conclusões: O uso de inferências em ontologias pode se dar de diversas formas, existindo uma variação na expressividade que os tipos de inferências possuem. Verificamos que as inferências podem agregar lógica às representações dos dados, visto que, a contextualização das informações com o uso de ontologias contém axiomas, aumentando a capacidade representacional das informações ao se utilizar inferências. Destaca-se a linguagem OWL como referência para enriquecimento e inserção de axiomas na construção das ontologias.
\end{abstract}

Palavras-chave: Web Semântica. Linked Data. Inferências. Ontologias. Sparql.

*Doutor em Ciência da Informação pela Universidade Estadual Paulista (Unesp - Marília). Professor da Universidade de São Paulo (USP). Professor do Programa de Pós-Graduação em Ciência da Informação da (Unesp-Marília). E-mail: santarem@usp.br

**Mestrando do Programa de Pós-Graduação em Ciência da Informação da Universidade Estadual Paulista (Unesp - Marília). E-mail: caio.coneglian@gmail.com

Pesquisa desenvolvida com o apoio do Conselho Nacional de Desenvolvimento Científico e Tecnológico (CNPq).

Inf. Inf., Londrina, v. 21, n. 2, p. 217 - 244, maio/ago., 2016.

http:www.uel.br/revistas/informacao/ 


\section{INTRODUÇÃO}

As tecnologias e os conceitos da Web Semântica têm levado os pesquisadores da área de Ciência da Informação a refletir acerca de seus objetos e tópicos de pesquisa. Dentre essas tecnologias, destacamos o uso dos principais padrões de metadados para descrição de objetos digitais, a linguagem eXtensible Markup Language (XML) e sua facilidade para interoperar dados, a linguagem Resource Description Framework (RDF) e sua capacidade de conectar recursos, as ontologias e suas linguagens de construção, como Web Ontology Language (OWL), que possibilita contextualizar e tornar computável a conceitualização de domínios e processos, o Protocol and RDF Query Language (SPARQL), capaz de interagir diretamente com os dados e tornar possível a recuperação da informação nesse contexto, e principalmente 0 conjunto de melhores práticas e materialização dessas tecnologias, o Linked Data.

Os textos clássicos que vêm estabelecendo os princípios e caminhos para Web Semântica, ontologias e mais recentemente sobre o Linked Data como "The Semantic Web: A new form of Web content that is meaningful to computers will unleash a revolution of new possibilities" de 2001, publicado por Berners-Lee, Hendler e Lassila; "The Semantic Web Revisited" de 2006, publicado por Shadbolt, Hall e Berners-Lee; "Understanding, building and using ontologies" de 1996, de Guarino; "Linked Data - The Story So Far" de 2009, publicado por Bizer, Heath e Berners-Lee e "Linked Data: Design Issues", de 2006, publicado por Berners-Lee, além de muitos outros papers que trabalham diretamente com a questão do significado no contexto da Web tem abordado dois conceitos fundamentais para efetiva consolidação da Web Semântica e também das melhores práticas para constituição do Linked Open Data, são eles a Serendipidade e a Inferência.

A serendipidade se refere a descobertas feitas ao acaso, capacidade que as tecnologias da Web Semântica e principalmente o Linked Data trazem à tona e possibilitam através da ligação semântica entre dados de fontes diversas espalhadas pelo mundo. Enquanto a inferência diz respeito a capacidade de se deduzir ou tomar decisões, baseadas na consolidação de 
José Eduardo Santarem Segundo; Caio Saraiva Coneglian

Web semântica e ontologias: um estudo sobre construção de axiomas e uso de inferências

uma verdade de uma proposição que não é conhecida, mas é tida a partir de sua relação direta com outras verdades existentes, podendo ser considerada uma das cerejas do "bolo de noiva da Web Semântica".

Ao nos depararmos com a inferência e a serendipidade que a Web Semântica e o Linked Data nos propõem, independente de dados científicos, percebe-se que é possível caminhar-se para uma seara de descobertas e contribuições surpreendentes nas mais variadas áreas do conhecimento.

Esta pesquisa tem como objeto principal a inferência no contexto das tecnologias inerentes a Web Semântica, e consequentemente ao Linked Data, entretanto a serendipidade, não discutida de forma conceitual e empírica neste momento, está indiretamente presente neste estudo.

A principal questão que permeia este trabalho é "há maneiras diferentes de pensar em inferência no contexto da Web Semântica?", entretanto junto a esta questão principal, há muitas outras relacionadas, portanto, pensemos também na questão "a inferência deve-se constituir dentro dos agentes de software que pretender recuperar dados em ambientes semânticos ou é papel das próprias ontologias já estabelecerem regras que proponham as inferências independente de qual seja o agente de busca?".

Essas questões em muitos casos estão diretamente ligadas a constituição de um ambiente semântico, na propositura de um dataset de dados baseado nos padrões do Linked Data, na formulação de ontologias e também na constituição de agentes de software, principalmente nos baseados no protocolo SPARQL.

Caracteriza-se, portanto, como objetivo deste trabalho: Identificar e analisar os modelos de inferência possíveis no contexto das tecnologias da Web Semântica. A metodologia utilizada consistiu-se em duas partes, sendo a primeira um levantamento bibliográfico e revisão de literatura para discussão do tema, e na segunda um estudo exploratório baseado em provas de conceito e testes de uso de inferências em aplicações distintas. 
José Eduardo Santarem Segundo; Caio Saraiva Coneglian

Web semântica e ontologias: um estudo sobre construção de axiomas e uso de inferências

\section{WEB SEMÂNTICA, ONTOLOGIAS, LINKED DATA E SPARQL}

A Web Semântica, proposta por Tim Berners-Lee, James Hendler e Ora Lassila, em artigo publicado na Scientific American em 2001 propõe, essencialmente, que se estruture os dados da Web de forma que eles possam ter significado e principalmente que se tornem passíveis de interpretação por máquinas, através de agentes computacionais. Os autores destacam as linguagens XML e RDF como essenciais para a consolidação da Web Semântica, assim como definem as ontologias como responsáveis por organizar o conhecimento neste novo paradigma da Web.

A XML é uma linguagem para representação sintática de recursos de maneira independente de plataforma. A linguagem XML permite agregar semântica aos documentos, deixando por conta de cada aplicação a interpretação da marcação atribuída a este conteúdo. Esta abordagem amplia significativamente as possibilidades do uso das linguagens de marcação, entre elas a capacidade de definir metadados - dados que descrevem dados (CAMPOS; SANTACHE; TEIXEIRA, 1999).

Segundo o World Wide Web Consortium (W3C), o RDF é uma linguagem de uso geral para representar informações na Web. O RDF tem como princípio fornecer interoperabilidade aos dados, de forma que possa contribuir com a recuperação de informações de recursos na Web. Um dos principais objetivos da linguagem RDF é justamente criar uma rede de informações a partir de dados distribuídos.

Construir uma rede de informações onde os nós estejam semanticamente ligados, formando um grande grafo global, com informações advindas de várias fontes diferentes ao redor do planeta, é o conceito central da chamada Web de Dados. Um grafo é um modelo matemático poderoso que pode ser aplicado na resolução de um conjunto de problemas. É composto por um conjunto de vértices e arestas/arcos (SANTAREM SEGUNDO, 2010).

Por meio do uso das tecnologias citadas, caracterizam-se as ontologias como um dos principais elementos da Web Semântica na construção de 
José Eduardo Santarem Segundo; Caio Saraiva Coneglian

Web semântica e ontologias: um estudo sobre construção de axiomas e uso de inferências

informações relacionadas que apresentem significado. Santarem Segundo (2015, p. 226) afirma que:

Utilizar ontologias é uma das maneiras de se construir uma
relação organizada entre termos dentro de um domínio,
favorecendo a possibilidade de contextualizar os dados,
tornando mais eficiente e facilitando o processo de
interpretação dos dados pelas ferramentas de recuperação da
informação.

Para Guarino (1998, p. 7, tradução nossa), ontologia é "uma maneira de se conceitualizar de forma explícita e formal os conceitos e restrições relacionados a um domínio de interesse”. Gruber (1993, p. 2, tradução nossa) define ontologias como uma "especificação explícita de uma conceituação". Uma conceituação pode ser representada como um conjunto de objetos, restrições, relacionamentos e entidades que se assumem necessárias em alguma área de aplicação.

Santarem Segundo e Coneglian (2015, p. 227) indicam que:

Para o uso como tecnologia da Web Semântica, entende-se as ontologias como: artefatos computacionais que descrevem um domínio do conhecimento de forma estruturada, através de: classes, propriedades, relações, restrições, axiomas e instâncias.

Para que se tornem efetivamente computacionais, as ontologias precisam passar de uma estrutura conceitual para implementação através de uma linguagem. Há um conjunto de linguagens que foram desenvolvidas ao longo dos anos para representação de ontologias. Algumas dessas linguagens como: Ontology Inference Layer (OIL), DARPA Agent Markup Language (DAML), depois DAML + OIL, constituíram um caminho até a chegada da Web Ontology Language (OWL).

A OWL é uma linguagem de marcação semântica para a definição, a instanciação, a publicação e a partilha de ontologias na World Wide Web. OWL é desenvolvida como uma extensão do vocabulário RDF e é proveniente de uma revisão das linguagens DAML + OIL (BECHHOFER et al., 2004). 
A linguagem OWL é reconhecida, atualmente, como o último padrão em linguagens para ontologia e recomendada como a principal linguagem para construção de ontologias, pelo consórcio W3C. (SANTAREM SEGUNDO, 2010, p. 127). A linguagem OWL oferece três sublinguagens, projetadas para uso de implementadores e comunidades específicas, que se apresentam a seguir em ordem de expressividade: OWL Lite, OWL DL e OWL Full.

Como já fora observado, um dos elementos que compõe, ou está diretamente ligado, as ontologias é a construção de axiomas que permitem a inferência. A inferência é citada em grande parte das pesquisas que tratam de Web Semântica como sendo o grande diferencial na construção de ambientes semânticos.

As regras que possibilitam a inferência dão mais qualidade a análise dos dados, visto que permitem conhecer novas relações dentro de um conteúdo e em alguns casos também permitem detectar possíveis inconsistências. Berners-Lee, Hendler e Lassila (2001, tradução nossa) dizem que "Para a web semântica funcionar, os computadores devem ter acesso a coleções estruturadas de informações e conjuntos de regras de inferência, que eles podem usar para conduzir o raciocínio automatizado".

\section{1 $\quad$ Linked Open Data e SPARQL}

Nos últimos anos vários elementos foram surgindo e ampliando o contexto da ideia original de Web Semântica de Berners-Lee. O W3C iniciou um processo de publicar, de efetivar e de disseminar um conjunto de tecnologias que foram se agregando em busca da Web Semântica ideal. Vários projetos ao redor do mundo também foram evoluindo de forma a constituir ambientes semânticos, tanto do ponto de vista de estrutura informacional quanto da possibilidade de recuperação semântica da informação. Dentro desse processo nasceu, também por iniciativa de Berners-Lee e sua equipe, 0 Linked Open Data.

Santarem Segundo (2015, p. 224) afirma que

Estruturar dados abertos de forma semântica não é apenas uma das formas de estabelecer a ligação entre o conceito de Dados Abertos e de Web Semântica, mas sim de estabelecer 
um modelo de estrutura de dados que favoreça o atendimento ao quinto princípio de dados abertos e também ao inciso da Lei de Acesso a Informação, que indicam a possibilidade dos dados serem processados por máquina, além da ligação entre informações de bases diferentes através de relacionamentos semânticos.

Essa característica torna os dados não apenas acessíveis e processáveis por máquinas, mas passíveis de processos de organização que podem facilitar a geração de novos dados, apresentação de resultados, relação com outros grupos de dados, aumento do conhecimento para tomadas de decisão, novos modelos de dados gerados a partir do relacionamento e cruzamento de dados de várias esferas governamentais, além da geração de novos modelos mentais de apresentação da informação de forma a facilitar o acesso dos dados pela sociedade civil. (SANTAREM SEGUNDO, 2015).

O Linked Open Data, que atualmente apresenta-se como a principal forma de materialização dos conceitos e tecnologias da Web Semântica, é um projeto, com um conjunto de normas a serem seguidas, que usa os mesmos princípios de ligação semântica da Web de Dados, entretanto tem particularidades específicas, indicando um grau de exigência maior na constituição de sua rede de interligações.

Segundo Heath e Bizer (2011, tradução nossa, n.p.), "o LOD [Linked Open Data] é um conjunto de melhores práticas para publicação e conexão de dados estruturados na Web, permitindo estabelecer links entre itens de diferentes fontes de dados para formar um único espaço de dados global".

Para Berners-Lee (2006, tradução nossa, n.p.):

\begin{abstract}
A Web Semântica não trata apenas de depósito de dados na web. Trata-se de fazer ligações, de modo que uma pessoa ou máquina possa explorar esse conjunto de dados. Com LOD [Linked Open Data], quando você tem um pouco de dados, você pode encontrar outros que estão relacionados.
\end{abstract}

Uma das chaves para recuperar o conteúdo do Linked Data, assim como de todos os dados ligados que estão disponíveis em ambientes semânticos, é o protocolo SPARQL. 
José Eduardo Santarem Segundo; Caio Saraiva Coneglian

Web semântica e ontologias: um estudo sobre construção de axiomas e uso de inferências

O SPARQL é um conjunto de especificações que fornecem linguagens e protocolos para consultar e manipular o conteúdo publicado em RDF na Web. O padrão compreende as seguintes especificações: uma linguagem de consulta para RDF; uma especificação que define uma extensão do SPARQL Query Language para executar consultas distribuídas em diferentes terminais SPARQL; uma especificação que define a semântica de consultas SPARQL sob regimes de vinculação, como RDF Schema, OWL, ou RIF; um protocolo que define os meios para a transmissão de consultas SPARQL arbitrárias e solicitações de atualização para um serviço de SPARQL; uma especificação que define um método de busca e descoberta e um vocabulário para descrever serviços SPARQL e um conjunto de testes, para avaliação da especificação SPARQL 1.1 (SPARQL, 2013).

O protocolo SPARQL é a base para a recuperação da informação em ambientes semânticos, entretanto a maximização de seu uso efetivo está ligada diretamente ao domínio da sua grande diversidade de funcionalidades.

Santarem Segundo (2014, p. 3870), fazendo uma abordagem sobre o uso do SPARQL no contexto do Linked Open Data, afirma que "é necessário transformar todo esse conjunto de informações em conhecimento útil e aplicável, de forma a mudar positivamente a vida das pessoas. Essa era a proposta inicial de Berners-Lee com a Web Semântica."

Desta forma, as inferências em ontologias podem agregar o processo de descoberta de dados pelos usuários, quando se trata do uso do Linked Data, e em quaisquer outras fontes de dados semanticamente relacionadas. As regras de inferência e os axiomas podem ser construídos de várias maneiras. Como esse é o objeto principal deste estudo, trataremos com maior ênfase no próximo tópico. 
José Eduardo Santarem Segundo; Caio Saraiva Coneglian

Web semântica e ontologias: um estudo sobre construção de axiomas e uso de inferências

\section{INFERÊNCIA, MOTOR DE INFERÊNCIA E LINGUAGENS PARA ONTOLOGIAS}

As inferências têm como sua base a dedução ou a tomada de decisão a partir de determinadas lógicas, traduzidas em expressões que são os chamados Axiomas. Desta maneira, no contexto computacional existem inúmeras formas matemáticas e algorítmicas para que estas inferências sejam aplicadas e gerem a tomada de decisão, como inferências a partir de lógicas Fuzzy e bayesianas. A partir da ideia de serem realizadas inferências com 0 uso de dados e de determinadas regras, é necessário que existam motores de inferências, para a geração das inferências em si.

Os motores de inferência são mecanismos capazes de atuarem seguindo lógicas pré-definidas. Inicialmente, os motores de inferência foram criados dentro dos estudos de Inteligência Artificial, sendo uma ferramenta com atuação em Sistemas Especialistas. Um Sistema Especialista pode ser entendido como uma aplicação criada para "[...] simular a ação de especialistas humanos, com o propósito de solucionar problemas específicos em um dado domínio." (BRASIL, 1999, p. 3).

Neste sentido, os motores de inferência são responsáveis por possibilitar que as expressões lógicas definidas possam gerar novos conhecimentos, visto que, são estes mecanismos que possibilitarão a deduções e a tomadas de decisões. Estes mecanismos necessitam de regras (axiomas) para realizar a inferência, sendo que existem diversas linguagens para a criação das regras.

No âmbito da Web Semântica, as ontologias são os principais elementos que embasam as lógicas de geração de inferências, pois estas representações fornecem elementos contendo axiomas. Esses axiomas são as bases lógicas para os motores de inferências, visto que os motores de inferências se utilizam dessas regras para executar as inferências nas estruturas das ontologias. Dentre os motores de inferências utilizados para lógicas em inferências e na Web Semântica, destacam-se ELK Reasoner, FaCT ++ Reasoner, Hermit Reasoner, Ontop Reasoner e Apache Jena, destacando que esses motores de inferência podem ser chamados de Semantic Reasoner. 
José Eduardo Santarem Segundo; Caio Saraiva Coneglian

Web semântica e ontologias: um estudo sobre construção de axiomas e uso de inferências

Os axiomas podem ser construídos em diversas linguagens lógicas. A Semantic Web Rule Language (SWRL), recomendada pelo W3C, é atualmente a que tem tido maior uso e relevância. A SWRL se baseia na linguagem OWL, utilizando o conjunto de axiomas desta linguagem para possibilitar a construção das regras lógicas. Dentre as regras do SWRL, destacam-se as propriedades "differentFrom", que tem como função a diferenciação de dois objetos e "sameAs" que indica que dois objetos são iguais. (HORROCKS et al., 2004).

Em síntese, a criação de regras construídas com SWRL contém duas partes essenciais, a primeira parte, uma ou um conjunto de condições, e a segunda parte que contém a inferência a ser executada caso a primeira parte da expressão seja verdadeira. No Quadro 1, é possível observar um exemplo de axioma, onde a primeira parte da expressão indica que se um elemento $A$ é subordinado a um elemento $B$ e se um elemento $B$ é subordinado a um elemento $\mathrm{C}$, infere-se que o elemento $\mathrm{A}$ é subordinado ao elemento $\mathrm{C}$, mesmo que essa afirmação não esteja descrita na ontologia. Essa relação descrita, é conhecida na construção de ontologias para caracterizar propriedades e relações chamadas de transitivas.

Quadro 1 - Exemplo de Axioma

subordinado $(A, B) E$ subordinado(B,C) $\Rightarrow$ subordinado $(A, C)$

Fonte: Elaborado pelos autores

A partir destas regras, o motor de inferências é capaz de verificar quais dados atendem as condições e poderão assim inserir os dados alterados de acordo com as lógicas existentes.

Uma outra maneira, talvez a mais rica delas, de constituir regras que permitam a inferência em ambientes semânticos é a utilização das possibilidades extras que a linguagem OWL oferece como diferencial em relação a outras linguagens para construção de ontologias. Algumas propriedades da linguagem OWL são capazes de embutir axiomas e definir enriquecimento semântico além da simples definição de características a um elemento.

Algumas dessas propriedades são: "owl:equivalentProperty", "owl:inverseOf", "owl:TransitiveProperty" e "owl:SymmetricProperty". 
José Eduardo Santarem Segundo; Caio Saraiva Coneglian

Web semântica e ontologias: um estudo sobre construção de axiomas e uso de inferências

A propriedade "owl:equivalenteProperty" indica que duas propriedades são equivalentes quando estas possuem o mesmo significado. Exemplo: Tanto faz relatar que um vinho é produzido por uma vinícola como afirmar que ele é fabricado por esta mesma vinícola.

A propriedade "owl:inverseOf" indica uma relação de inversão. A caracterização se dá quando um determinado elemento $A$ se relaciona com 0 elemento $\mathrm{B}$ através da propriedade $\mathrm{P}$, e se essa propriedade $\mathrm{P}$ é inversa de $P 2$, isso implica que o elemento $B$ se relaciona com $A$ através de P2. Exemplo: se um Vinho é "Produzido" por uma Vinícola e a propriedade "Produzido" é inversa a propriedade "Produz", isso indica que a Vinícola "produz" o Vinho.

A propriedade "owl:TransitiveProperty", indica que se um elemento A tem uma relação $X$ com o elemento $B$ e $B$ tem uma relação $X$ com o elemento $C$, logo A tem uma relação $X$ com $C$.

A propriedade "owl:SymmetricProperty", indica uma relação de simetria entre dois elementos, indicando que se um elemento $A$ está relacionado a $B$, isso implica que $B$ também está relacionado a $A$.

Essas propriedades, assim como outras características da linguagem OWL tornam sua expressividade semântica superior a outras linguagens para construção de ontologias, exigindo da equipe de construção de ontologias um conhecimento mais aprofundado acerca do domínio e também das características da linguagem para que se possa explorar ao máximo essas possibilidades. Ao usar essas características da linguagem OWL na construção de ontologias, as inferências são possíveis com utilização básica da linguagem SPARQL no momento da consulta. As implementações referentes a essas técnicas serão apresentadas no tópico seguinte.

\section{RESULTADOS E DISCUSSÃO: CONSTRUÇÃO DE AXIOMAS E EXECUÇÃO DE INFERÊNCIAS}

O uso de lógicas e axiomas em ambientes computacionais aprimoram os processos de inteligência e de descoberta das informações, uma vez que permite com que os dados possam realizar e conter inferências. No contexto da Web Semântica e o do Linked Open Data, o uso de lógica pode agregar mais 
José Eduardo Santarem Segundo; Caio Saraiva Coneglian

Web semântica e ontologias: um estudo sobre construção de axiomas e uso de inferências

relevância no momento de contextualizar e de explorar um determinado domínio, permitindo com que a descrição semântica dos dados contenha também meios que possibilite os processos de inferência.

Desta forma, as ontologias se mostram como o principal instrumento para a aplicação de inferências, pois devido as ontologias apresentarem estruturas semânticas e propriedades com axiomas, muitas vezes a lógica para a aplicação das inferências se encontram nas próprias estruturas da ontologia. Uma outra característica das ontologias que favorece o uso de inferências, é que a ontologia é uma estrutura formal, que permite com que algoritmos computacionais consigam compreender os dados ali contidos.

Dentro do Linked Open Data, há diversas ontologias em OWL que estruturam os dados, além da utilização de diversas propriedades semânticas para realizar a contextualização das informações. Assim, os agentes computacionais ao explorarem os datasets, se encontram frente a estes elementos, que fornecem lógicas para uma compreensão mais adequado dos dados. Partindo deste princípio, a utilização das lógicas e das inferências das ontologias permitem com que a abrangência das consultas dos dados seja mais assertiva.

Contudo, há diversas formas de serem construídas as inferências, existindo uma variação nos níveis de lógica e de semântica em cada uma destas maneiras. Este é o objeto principal desta pesquisa e partir destas condições, buscou-se identificar os tipos de inferências existentes, bem como suas características realizando testes como prova de conceito de cada tipo de inferência.

Primeiramente é necessário relatar um elemento essencial na questão da recuperação e apresentação dos dados e que, portanto, apresenta um papel fundamental na discussão sobre inferências, que é o protocolo SPARQL. O SPARQL tem uma função chave na questão da inferência por estar posicionado na fase final da recuperação dos dados de uma ontologia ou de conjunto de dados que utiliza ontologias. Desta forma, este protocolo poderá ser aplicado para a realização de inferências em conjuntos de dados ou 
José Eduardo Santarem Segundo; Caio Saraiva Coneglian

Web semântica e ontologias: um estudo sobre construção de axiomas e uso de inferências

somente para apresentar dados cuja as inferências se deram na sua própria estrutura.

Por meio desta constatação, verifica-se que há duas formas principais de se inferir informações sobre os dados, a primeira ocorre por meio de construções utilizando-se da flexibilidade e complexidade do protocolo SPARQL, de forma que o protocolo em sua execução insira os dados baseados em inferências nos resultados obtidos e a segunda quando a construção do SPARQL não tem a preocupação em construir a lógica de inferência, visto que os axiomas e as lógicas de inferência estão completamente contidas nas estruturas dos dados. Na sequência serão exploradas essas duas formas e as variações existentes em cada uma delas.

As inferências construídas com o uso do SPARQL apresentam como característica central a inserção da lógica na própria consulta. Assim, o usuário, em geral um programador ou usuário de alto nível em linguagens, no momento de construir uma consulta deverá ter conhecimento do conjunto de dados e da ontologia que está trabalhando. A inserção de lógica dentro do SPARQL é possível devido ao ao protocolo ser baseado no RDF, permitindo com que sejam explicitados e identificadas relações contidas na base de dados e que o usuário conseguiu compreender a partir de uma análise. Ademais, a inserção de inferências dentro do SPARQL poderá ter dois níveis de semântica, ocorrendo de acordo com o grau de uso das propriedades do RDF e do OWL que a consulta utiliza para realizar as suas inferências.

Neste sentido, o primeiro nível tem como característica central a não utilização das propriedades semânticas do OWL, sendo a inferência construída de acordo com as lógicas inseridas na consulta, dado um conhecimento prévio do usuário acerca da base de dados/ontologia a ser consultada. Para comprovar a veracidade desta afirmação, realizou-se um teste em que fora utilizada uma estrutura de dados simples que não contém as propriedades semânticas do OWL. Esta estrutura apresenta um conjunto de dados hierárquicos, com lógica familiar, que demonstra as relações de descendência e ascendência. Na estrutura, não há informações acerca de outros graus de parentescos como por exemplo, tio e tia, irmão ou primo, como apresentado na 
José Eduardo Santarem Segundo; Caio Saraiva Coneglian

Web semântica e ontologias: um estudo sobre construção de axiomas e uso de inferências

Figura 1, que contém a esquerda os dados em esquema de grafos e a direita estes mesmos dados na notação N3.

Figura 1 - Conjunto de Dados - Inferência 1

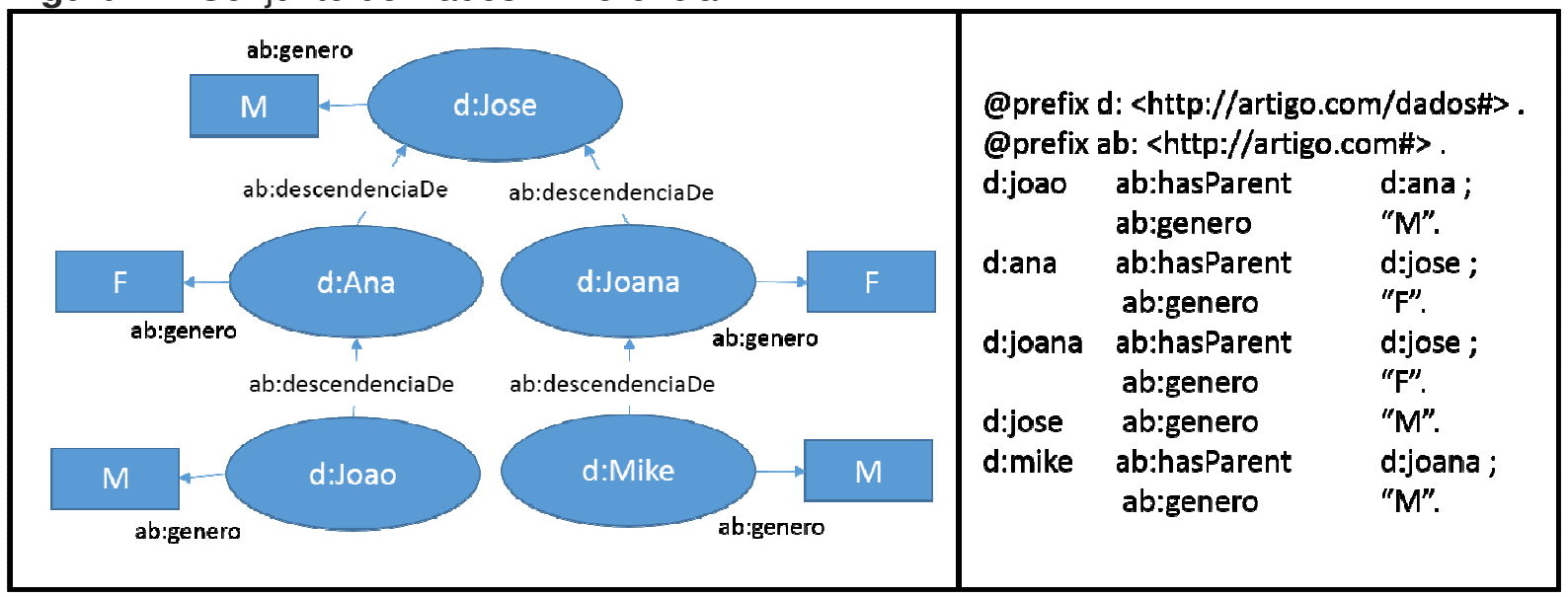

Fonte: Elaborado pelos autores.

A partir dos dados apresentados na Figura 1, é possível realizar-se inferências que permitam definir outros tipos de parentescos além dos já apresentados. Como não há quaisquer relações que apontem esses graus de parentesco é necessário que tal informação seja inferida por meio do uso do SPARQL.

A Figura 2 demonstra o teste realizado que em síntese encontra o grau de parentesco tia, demonstrando uma pessoa e sua respectiva tia. A esquerda da Figura 2 está contido a consulta SPARQL com a lógica e na parte da direita apresenta-se os resultados obtidos a partir desta consulta.

Figura 2 - Consulta - Inferência 1

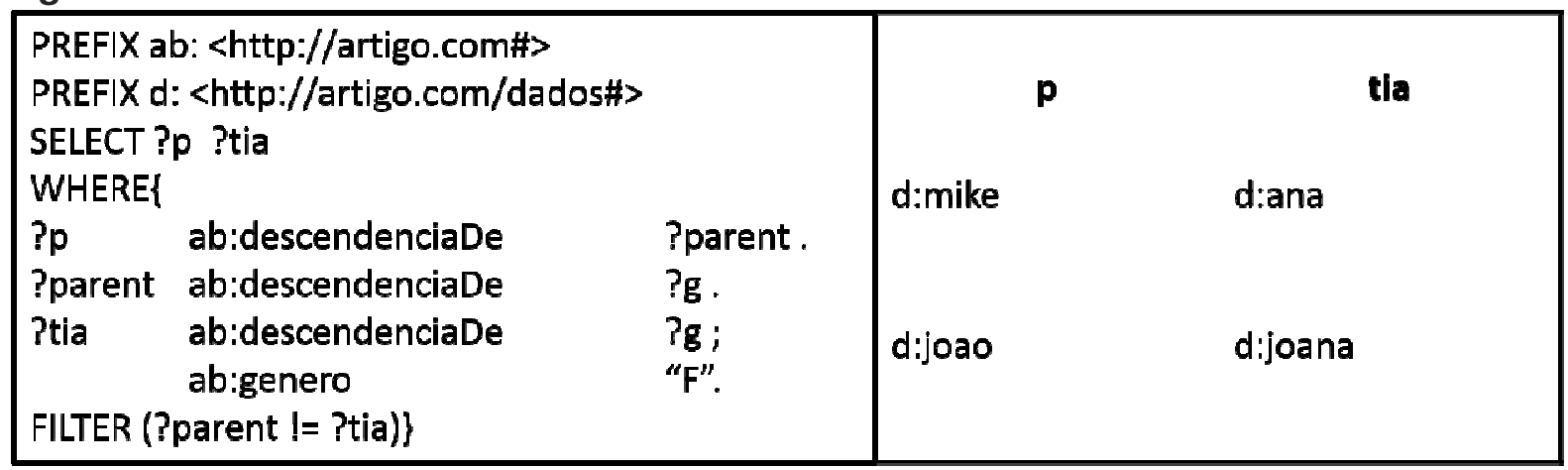

Fonte: Elaborado pelos autores. 
José Eduardo Santarem Segundo; Caio Saraiva Coneglian

Web semântica e ontologias: um estudo sobre construção de axiomas e uso de inferências

A lógica para encontrar o grau de parentesco tia, necessita que o sobrinho e a tia tenham um ponto comum que seria uma pessoa posicionada acima com, no mínimo, dois graus de descendência (avô ou avó) para o sobrinho e que tenha um grau de descendência com a tia, sendo que é necessário que a tia seja do gênero feminino, bem como a tia não esteja em sua linha ascendente direta, ou seja, não ser o pai ou a mãe. Essa lógica pensada por um usuário pode ser traduzida em uma consulta SPARQL, a partir da definição de relações, como a identificação do ponto comum, que seria o avô ou avó, traduzido na consulta com a variável "?g" e a definição do gênero feminino "F".

Os resultados obtidos demonstram que a consulta SPARQL consegue realizar a inferência e localizar uma pessoa e a sua respectiva tia. Vale ressaltar que, para que um usuário localize a tia por meio do esquema de grafo, utiliza-se um processo simples devido aos conhecimentos prévios que esse usuário possui, contudo para que um programa computacional faça essa inferência, é necessário que essas regras sejam definidas computacionalmente. A construção dessa consulta SPARQL deverá refletir as lógicas existentes para que o computador apresente os resultados corretos.

Ainda tratando de lógicas dentro da consulta SPARQL, há um segundo nível de inferências dentro das consultas, que tem como característica a utilização das propriedades do OWL para a inserção de lógica. Este nível avança consideravelmente na semântica inserida nas inferências, pois abrange os principais elementos das ontologias OWL no que diz respeito a contextualização de um domínio. Desta forma, ao contrário da consulta anterior, a inferência neste nível usa os elementos semânticos dentro da ontologia, tornando as lógicas mais abrangentes, ao passo que o usuário ao criar a consulta não necessita conhecer todas as características daqueles dados, porém deve ter um conhecimento mais amplo da linguagem OWL e de suas propriedades.

Para demonstrar como estas inferências são realizadas, utilizou-se um exemplo de fronteiras entre países, em que a ontologia construída apresenta 
José Eduardo Santarem Segundo; Caio Saraiva Coneglian

Web semântica e ontologias: um estudo sobre construção de axiomas e uso de inferências

algumas propriedades semânticas. A Figura 3 ilustra o conjunto de dados utilizados para a realização deste segundo tipo de inferência.

Figura 3 - Conjunto de Dados - Inferência 2

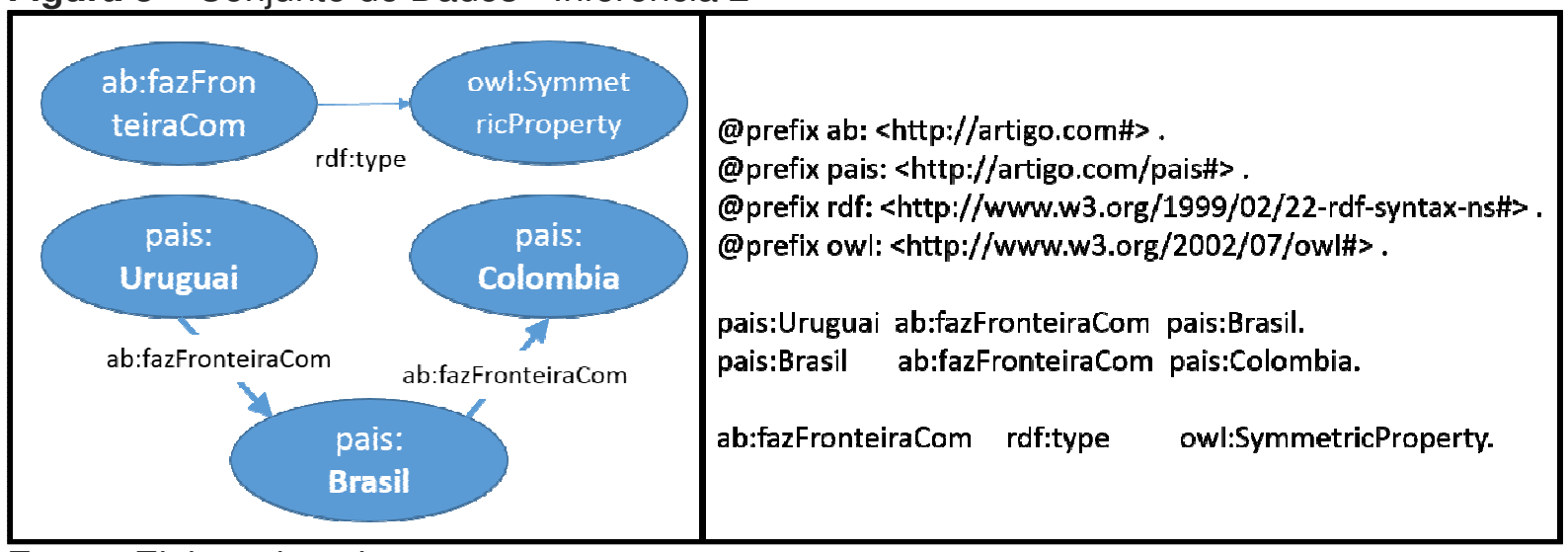

Fonte: Elaborado pelos autores.

O conjunto de dados apresentados na Figura 3, contém dois tipos de informações, a primeira indica os relacionamentos entre três países: o objeto "pais:Urugual" tendo um relacionamento do tipo "ab:fazFronteiraCom" com o objeto "pais:Brasil", que por sua vez possui o relacionamento "ab:fazFronteiraCom" com "pais:Colombia". O segundo tipo de informação existente na ontologia é que a propriedade "ab:fazFronteiraCom" é do tipo simétrico, indicando que se o país $A$ faz fronteira com o país $B$, logo o país $B$ faz fronteira com o país A. Tal inferência é elementar para um usuário que possui noções acerca da geografia dos países, porém necessita estar descrito na ontologia para que agentes computacionais possam compreender tal informação.

Desta forma, uma consulta SPARQL que recupere os objetos que possui a relação "ab:fazFronteiraCom" com o "pais:Brasil" recuperaria somente o "pais:Uruguai", pois não levaria em conta que esta propriedade é do tipo simétrica. Partindo disso, é necessário que sejam feitas inferências na consulta, que leve em consideração caso a propriedade fosse simétrica, como a consulta ilustrada na Figura 4. 
José Eduardo Santarem Segundo; Caio Saraiva Coneglian

Web semântica e ontologias: um estudo sobre construção de axiomas e uso de inferências

Figura 4 - Consulta - Inferência 2

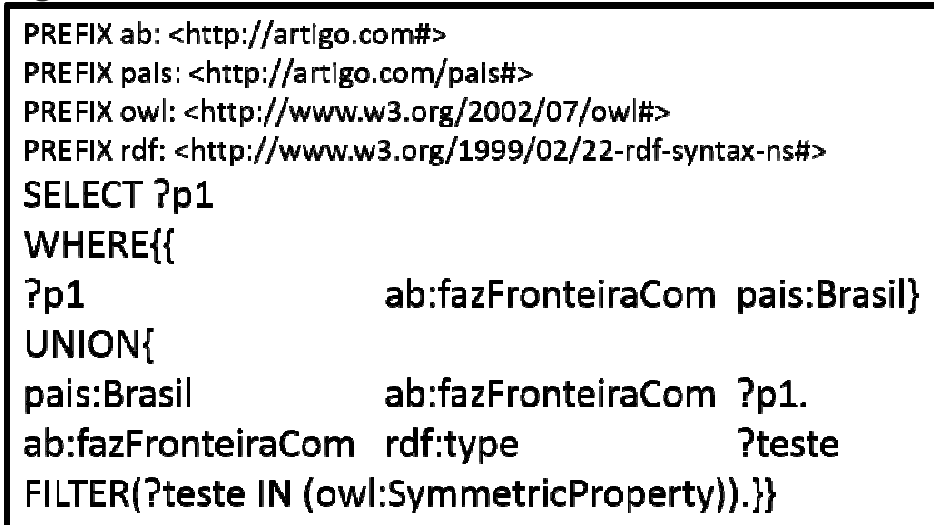

\begin{tabular}{|l|}
\hline \multicolumn{1}{|c|}{$\mathbf{p 1}$} \\
pais:Uruguai \\
\hline pais:Colombia \\
\hline
\end{tabular}

Fonte: Elaborado pelos autores.

A consulta demonstrada na Figura 4, contém elementos que permitem a realização da inferência, em que existe uma união entre todos os objetos que possuam uma ligação do tipo "ab:fazFronteiraCom" com "pais:Brasil" e todos os objetos que possuem a relação simétrica, sendo os objetos que o "pais:Brasil" tem relação por meio de "ab:fazFronteiraCom".

Há, ainda, a inserção de uma lógica no filtro, que define a realização dessa união somente se a relação "ab:fazFronteiraCom" for do tipo simétrico, ou seja, caso não tivesse sido definido que esta relação possuía a propriedade simétrica, pela consulta SPARQL não seria recuperado objetos pela união realizada na consulta. Assim, a consulta SPARQL apresenta inferências no que trata da utilização de uma propriedade do OWL, de modo que, todas as consultas que recuperam também as relações simétricas possuirão sintaxe semelhante.

Os dois níveis apresentados têm como semelhança o uso do SPARQL com construções complexas. Dependendo do usuário que constrói as consultas para tornar possível a lógica das inferências, sendo que há a necessidade de que em toda consulta realizada, o usuário reflita e explicite no SPARQL as lógicas, e caso uma consulta não utilize estas lógicas, a inferência não acontecerá. Esses níveis dependem excessivamente do conhecimento do usuário acerca dos conjuntos de dados e das ontologias a serem consultadas. Partindo disto, há uma segunda forma de realizar as inferências, em que as lógicas não estarão contidas dentro do SPARQL, e sim nas próprias estruturas da ontologia. Nesta segunda forma, os motores de inferência são os 
José Eduardo Santarem Segundo; Caio Saraiva Coneglian

Web semântica e ontologias: um estudo sobre construção de axiomas e uso de inferências

responsáveis por enriquecer o conjunto de dados. Os motores de inferência têm como função modificar as estruturas da ontologia atendendo à determinadas lógicas.

Esta segunda forma apresenta novamente dois níveis de inferências. $O$ primeiro nível tem como característica a definição de lógicas e de axiomas, que alteram os dados contidos na ontologia. Enquanto o segundo nível tem como princípio a utilização das propriedades semânticas e axiomas do próprio OWL. Da mesma maneira que na primeira forma de construir inferências, o primeiro nível apresenta uma abrangência limitada, enquanto o segundo permite inferências mais complexas e padronizadas. Na sequência demonstramos as características e as diferenças destes dois níveis.

O primeiro nível utiliza como essência da inferência a geração de regras, que contém axiomas lógicos. Tais axiomas expressam sentenças que tornamse ativas por meio do motor de inferência.

Para demonstrar o funcionamento desta terceira variedade de inferências, realizou-se um teste que utiliza uma ontologia semelhante a demonstrada na Figura 1, que ilustra as relações hierárquicas entre pessoas, gerando uma relação chamada de tia, com as mesmas regras contidas na ontologia. Basicamente, o princípio lógico da relação é o mesmo apresentado no primeiro caso de inferência, porém utilizando regras, que após a execução do motor de inferência, alteram a ontologia. A Figura 5 ilustra este terceiro tipo de inferência, contendo a regra criada na parte superior e ao centro apresentase a nova estrutura da ontologia depois de executar o motor de inferência, sendo possível identificar a alteração dos dados de acordo com as regras estabelecidas, que pode ser verificado pela inserção da relação "ab:tia" tanto no grafo quanto na notação N3. 
José Eduardo Santarem Segundo; Caio Saraiva Coneglian

Web semântica e ontologias: um estudo sobre construção de axiomas e uso de inferências

Figura 5 - Conjunto de Dados e Regras - Inferência 3

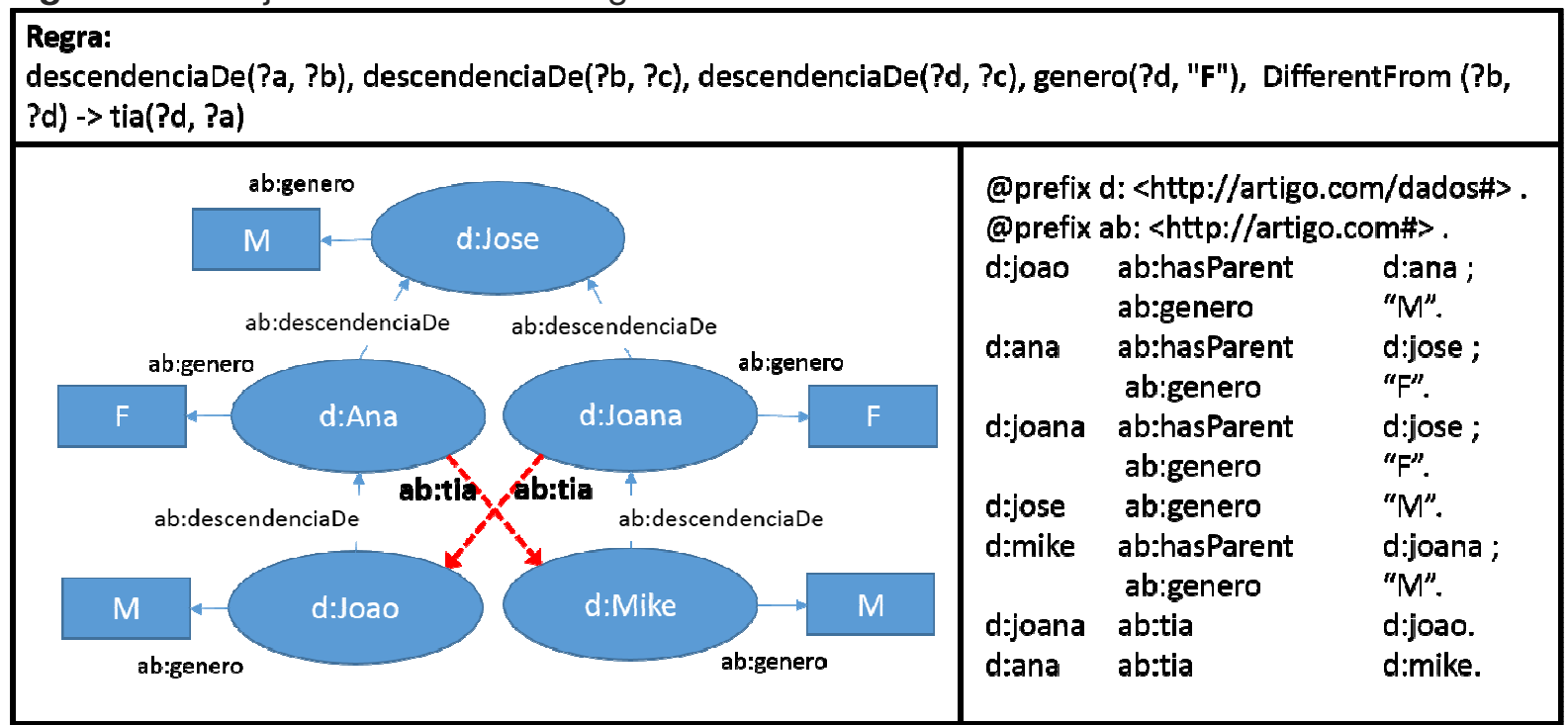

Fonte: Elaborado pelos autores.

A partir da Figura 5, verifica-se que as regras descritas alteram a estrutura dos dados, explicitando as novas relações criadas. Destaca-se que a estrutura original é a mesma da Figura 1, entretanto o motor de inferência utiliza-se da regra criada para incrementar o conjunto de dados com a nova relação criada. Vale destacar que no grafo da Figura 5, as únicas mudanças que foram realizadas são as linhas pontilhadas, contendo a legenda "ab:tia". Desta forma, no processo de recuperação dos dados, a consulta SPARQL não contém a lógica para identificar a relação tia, pois tal relação já estará inserida na própria ontologia, ficando na consulta somente a identificação da própria relação do tipo "ab:tia", recuperando os objetos que possuem a relação já estabelecida, como indicado pela Figura 6.

Figura 6 - Consulta - Inferência 3

\begin{tabular}{|c|l|l|}
\hline $\begin{array}{c}\text { PREFIX ab: <PREFIX ab: <http://artigo.com\#> } \\
\text { SELECT Ptia Psobrinho } \\
\text { WHERE \{ ?tia ab:tia ?sobrinho }\}\end{array}$ & toana & \multicolumn{1}{|c|}{ sobrinho } \\
\cline { 2 - 3 } & Ana & Joao \\
\hline
\end{tabular}

Fonte: Elaborado pelos autores.

As regras inserem uma gama de possibilidades às ontologias, permitindo com que regras de inferência insiram novos relacionamentos e caracterize de forma mais rica um modelo de dados. Contudo, as regras 
José Eduardo Santarem Segundo; Caio Saraiva Coneglian

Web semântica e ontologias: um estudo sobre construção de axiomas e uso de inferências

estabelecidas, como relatado, não são realizados dentro da linguagem OWL, tornando o processo de inferência limitado, pois está sujeito a capacidade de interpretação dos motores de inferência, uma vez que alguns motores podem não reconhecer determinadas linguagens para a construção de regras.

Assim, a quarta forma de utilização de inferências em ontologias se mostra como o nível que agrega maior semântica e representatividade, devido utilizar as próprias características do OWL para a identificação das regras e lógicas do modelo. Observa-se que OWL é a linguagem mais indicada para construir ontologias. A ideia central deste nível de inferência é que as características semânticas definidas pelas propriedades do OWL, sejam interpretadas pelo motor de inferências, e no momento de realizar a recuperação dos dados, sejam apresentadas as informações com as inferências realizadas.

Para demonstrar as características desse tipo de inferência, utilizou-se o mesmo exemplo apresentado na Figura 3, em que se usa a propriedade simétrica para realizar a recuperação dos dados. Entretanto na Figura7 é possível identificar que a propriedade "ab:fazFronteiraCom" é do tipo "owl:SymmetricProperty", utilizando-se da riqueza semântica e de implementação que a linguagem OWL oferece. Neste sentido, ao se realizar a recuperação, a consulta SPARQL deverá identificar que por ser uma relação simétrica, caso o inverso de uma relação de um objeto relacionado com outro objeto também é verdadeiro, devendo assim ser recuperado. Porém, no caso desta inferência a consulta SPARQL não terá a lógica que interprete que essa consulta é simétrica, e sim o motor de inferências que fará esse trabalho, de forma que, a consulta SPARQL retornará todos os resultados com a lógica tratada. A Figura 7 apresenta a demonstração dos dados de inferência do exemplo relatado. 
José Eduardo Santarem Segundo; Caio Saraiva Coneglian

Web semântica e ontologias: um estudo sobre construção de axiomas e uso de inferências

Figura 7 - Dados - Inferência 4

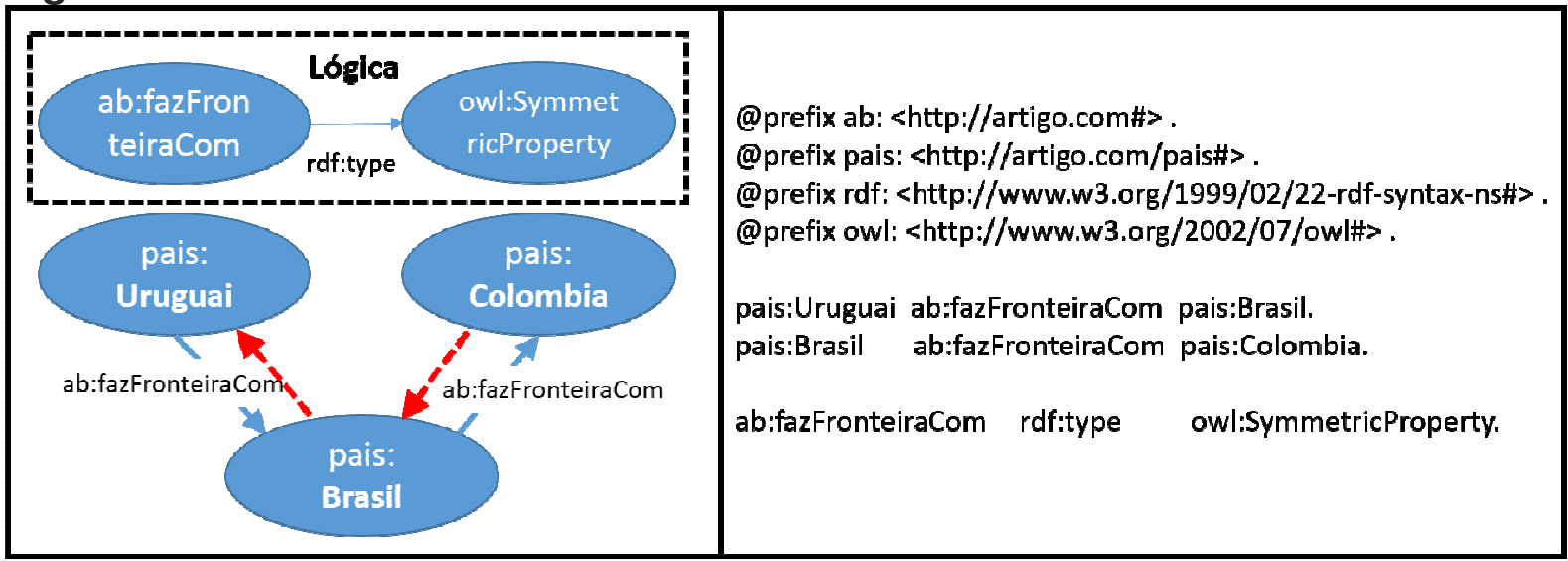

Fonte: Elaborado pelos autores.

A ilustração do grafo apresentado na Figura 7, indica as mesmas relações destacadas na Figura 3, com a inserção das relações estabelecidas com a lógica da relação simétrica, destacadas pelas linhas pontilhadas. No entanto, vale destacar que a relação simétrica criada não se mostra representada na notação $\mathrm{N} 3$, isto porque a ontologia em si não sofre alterações, somente há a interpretação pelo motor de inferências, que fornecerá tais informações no momento da realização da consulta SPARQL, indicada pela Figura 8.

Figura 8 - Consulta - Inferência 4

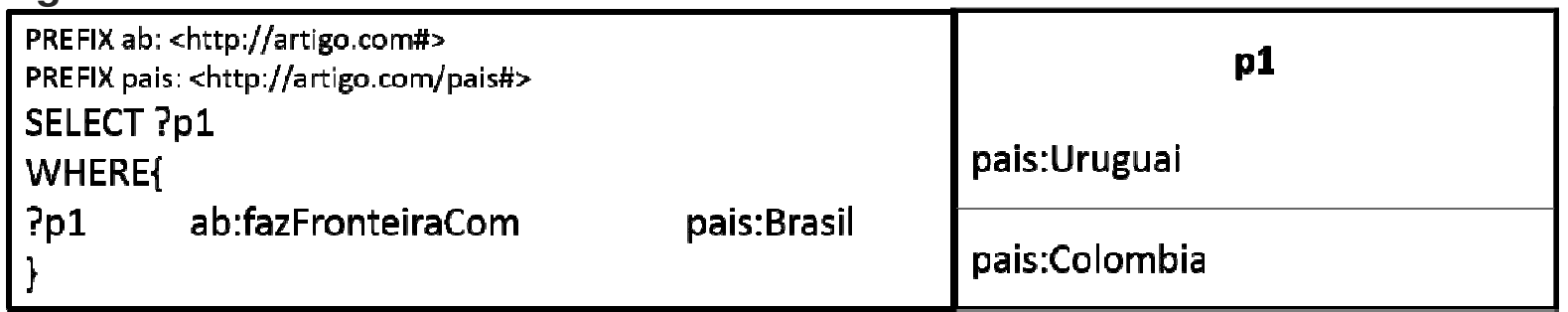

Fonte: Elaborado pelos autores.

Um outro destaque diz respeito a consulta SPARQL, contida parte esquerda da Figura 8, em que não há a inserção da lógica da inferência, e sim somente a definição da própria relação que deveria ser recuperada, e na parte direita, demonstra-se que os resultados recuperados foram obtidos a partir da inferência, pois a relação "pais:Brasil" faz fronteira com "pais:Colombia" não obedece sintaticamente a relação construída na consulta SPARQL, porém 
José Eduardo Santarem Segundo; Caio Saraiva Coneglian

Web semântica e ontologias: um estudo sobre construção de axiomas e uso de inferências

obedece semanticamente, devido a definição da propriedade simétrica, e a utilização desta pelo motor de inferências.

Assim este nível se mostra como o mais elaborado e que contém maior expressividade, pois um agente computacional ou um usuário ao realizar uma consulta em ontologias e bases de dados, não necessitará saber se há a definição das propriedades do OWL, como simétrico ou transitivo, devendo realizar somente uma consulta simples, que recuperará os dados corretamente. Neste nível, a responsabilidade da lógica deixa de ser do usuário ao realizar a consulta, e passa a ser do criador da ontologia a princípio e de um motor de inferências que deverá realizar a interpretação das propriedades existentes nas ontologias.

Vale destacar que, devido as diversas propriedades que o OWL possui, se tornaria extremamente complexo construir consultas SPARQL que contém as lógicas de interpretação de todas elas, tornando o uso do motor de inferências a melhor opção nestes casos, além de tornar a navegação dos agentes computacionais mais simples e livre de detalhes exclusivos de cada base de dados e ontologias. Necessário dar ênfase neste ultimo nível de inferência à melhora do processo de descoberta das informações, efetivando a serendipidade, principalmente quando se dão essas construções nos datasets do Linked Data.

Buscando resumir os resultados obtidos a partir da análise e identificação desses quatro tipos de inferências, criou-se o Quadro 2, que sintetiza as tais características. A primeira coluna do Quadro 2 indica qual a atuação do SPARQL com relação a presença de lógicas, a segunda coluna descreve o tipo de inferência descrito e a terceira coluna apresenta características destas inferências.

Inf. Inf., Londrina, v. 21, n. 2, p. 217 - 244, maio/ago., 2016. 
José Eduardo Santarem Segundo; Caio Saraiva Coneglian

Web semântica e ontologias: um estudo sobre construção de axiomas e uso de inferências

Quadro 2 - Tipos de Inferências em Ontologias

\begin{tabular}{|c|c|c|}
\hline SPARQL & $\begin{array}{l}\text { Tipo de } \\
\text { Inferência }\end{array}$ & Características \\
\hline \multirow[t]{2}{*}{$\begin{array}{l}\text { SPARQL } \\
\text { com lógica } \\
\text { para } \\
\text { inferência }\end{array}$} & $\begin{array}{l}\text { Inferências sem } \\
\text { utilizar os recursos } \\
\text { do OWL }\end{array}$ & $\begin{array}{l}\text { Neste tipo de inferência, a lógica deverá ser } \\
\text { definida totalmente na consulta SPARQL, } \\
\text { devendo o criador da consulta conhecer a } \\
\text { estrutura da ontologia e criar formas de } \\
\text { apresentar os resultados obtidos de modo a } \\
\text { obter resultados lógicos de uma sentença. }\end{array}$ \\
\hline & $\begin{array}{ll}\text { Inferências } & \\
\text { utilizando } & \text { as } \\
\text { propriedades } & \text { do } \\
\text { OWL } & \end{array}$ & $\begin{array}{l}\text { Remete a construção de inferências a partir } \\
\text { das propriedades do OWL (como simetria e } \\
\text { transitividade), em que a consulta SPARQL } \\
\text { deverá ser construída analisando se as } \\
\text { propriedades contêm alguma característica } \\
\text { semântica do OWL. }\end{array}$ \\
\hline \multirow[t]{2}{*}{$\begin{array}{l}\text { SPARQL } \\
\text { sem lógica } \\
\text { para } \\
\text { inferência }\end{array}$} & $\begin{array}{lr}\text { Criação } & \text { de } \\
\text { inferências } & \text { por } \\
\text { meio de regras } \\
\text { lógicas definidas } \\
\text { em um motor de } \\
\text { inferências }\end{array}$ & $\begin{array}{l}\text { No momento da criação de uma ontologia é } \\
\text { possível definir regras que realizará } \\
\text { adequações ao código OWL, de forma a a } \\
\text { atender as lógicas inseridas. Um motor de } \\
\text { inferências irá alterar a ontologia segundo as } \\
\text { regras definidas. Em geral as regras são } \\
\text { construídas utilizando-se a linguagem SWRL. }\end{array}$ \\
\hline & $\begin{array}{l}\text { Inferências } \\
\text { realizadas por um } \\
\text { motor de } \\
\text { inferências a partir } \\
\text { das propriedades } \\
\text { do OWL }\end{array}$ & $\begin{array}{l}\text { A utilização de um motor de inferências } \\
\text { permite com que inferências sejam realizadas } \\
\text { de forma automática a partir da definição das } \\
\text { propriedades semânticas do OWL. Desta } \\
\text { forma, a consulta SPARQL é simples, } \\
\text { entretanto recupera os dados com as } \\
\text { inferências devido ao motor de inferência } \\
\text { interpretar as propriedades do OWL. }\end{array}$ \\
\hline
\end{tabular}

Fonte: Elaborado pelos autores. 
José Eduardo Santarem Segundo; Caio Saraiva Coneglian

Web semântica e ontologias: um estudo sobre construção de axiomas e uso de inferências

A síntese apresentada no Quadro 2 indica quatro formas de realizar inferências, que devem ser utilizadas de acordo com as necessidades do projeto e das características dos conjuntos de dados disponíveis.

\section{CONSIDERAÇÕES FINAIS}

A Web Semântica, suas tecnologias e a materialização de suas propostas na constituição do Linked Data são uma realidade. Isso tem mudado a maneira de realizar pesquisa na Ciência da Informação moderna.

Dentre as abordagens da Web Semântica e principalmente do Linked Data ressalta-se que a construção de ontologias vem ganhando destaque, principalmente porque tem se tornado um dos pontos que ainda carecem de muita pesquisa, tanto do ponto de vista da questão tecnológica quanto de metodologias para que seja possível constituir modelos computacionais que realmente reflitam os conceitos de uma área ou de um domínio do conhecimento.

A questão da inferência, por diversas vezes citadas em papers tido como referência em Web Semântica, está totalmente inserida no contexto da construção das ontologias e influenciam diretamente a serendipidade no mar de conhecimento que tem se tornado o Linked Data e todos os outros modelos de dados ligados que vem se constituindo.

Para que as inferências sejam possíveis do ponto de vista computacional, é necessário que sejam construídos regras e axiomas que conduzam os agentes computacionais a tomarem decisões acerca de informações que não estão explicitadas em conjuntos de informações, mas que certamente são passíveis de dedução.

Nos testes de implementação, apresentou-se quatro formas principais de modelos que podem ser utilizados para que aconteça a inferência. Dentre eles destacam-se os modelos que necessitam de atuação efetiva do programador ou agente computacional no momento de construir a consulta utilizando a linguagem SPARQL. Observou-se que esse modelo deixa para o agente computacional a tarefa de realizar as inferências, sendo uma opção que permite ao agente tomar suas próprias decisões, mas por outro lado exige que

Inf. Inf., Londrina, v. 21, n. 2, p. 217 - 244, maio/ago., 2016. 
José Eduardo Santarem Segundo; Caio Saraiva Coneglian

Web semântica e ontologias: um estudo sobre construção de axiomas e uso de inferências

o agente computacional, ou o programador responsável por ele, tenha conhecimento aprofundado do conjunto de dados e principalmente da ontologia na qual será feita a recuperação da informação.

Por outro lado, observou-se que a os modelos que não exigem que as inferências estejam explicitadas pela linguagem SPARQL conduzem os agentes computacionais a uma recuperação da informação mais rica e principalmente que reflete mais precisamente as relações que podem ocorrer em conjuntos de dados do ponto de vista de quem publicou a ontologia, ou mais exatamente de quem planejou a ontologia a ser utilizada.

Esses modelos que não exigem tanto da linguagem SPARQL e principalmente do agente computacional, acontecem pela criação de axiomas através da linguagem SWRL com o uso posterior de motores de inferência, assim como na construção de ontologias utilizando-se da riqueza das propriedades diferenciadas da linguagem OWL.

Apesar do modelo de construção de axiomas pela linguagem SWRL ser muito utilizado, torna-se uma tarefa a mais para a equipe que está construindo a ontologia, além disso, torna-se um adendo a ontologia, que precisa ser trabalhado quase sempre como um elemento externo.

Por fim, considera-se que o modelo mais adequado para construção de ontologias do ponto de vista da realização de inferências é justamente o uso máximo das qualidades da linguagem OWL, que facilitam o trabalho do agente computacional, assim como tornam os axiomas disponíveis sem que seja necessário criar um conjunto de regras adicionais para a ontologia a ser utilizada. Esse é o modelo mais adequado quando vai se iniciar ou planejar um projeto de disponibilização de dados.

Ressalta-se que em muitas oportunidades o conjunto de dados já está posto, sem regras de inferência na sua estrutura, havendo uma necessidade latente de realizar as inferências ao consultar esses dados. Nesse caso é imprescindível conhecimento acerca do conjunto de dados e principalmente dos recursos que o protocolo SPARQL tem a oferecer. 


\section{REFERÊNCIAS}

BECHHOFER, S. et al. OWL Web Ontology Language reference. 2004. Disponível em: < http://www.w3.org/TR/owl-ref/>. Acesso em: 22 jun. 2016.

BERNERS-LEE T.; HENDLER, J.; LASSILA, O. The semantic web. Scientific American, New York, v. 5, May 2001.

BERNERS-LEE, T. Linked Data: Design Issues 2006. Disponível em <http://www.w3.org/Designlssues/LinkedData.html> Acesso em: 10 jun. 2016.

BIZER, C.; HEATH, T.; BERNERS-LEE, T. Linked data - The story so far. International Journal on Semantic Web and Information Systems, v. 5, n. 3, p. 22, 2009.

BRASIL, L. M.. Proposta de arquitetura para sistema especialista híbrido e a correspondente metodologia de aquisição do conhecimento. 1999. $256 \mathrm{f}$. Tese (Doutorado em Engenharia Elétrica) - Universidade Federal de Santa Catarina, Florianópolis. $1999 . \quad$ Disponível em: <https://repositorio.ufsc.br/bitstream/handle/123456789/81196/137930.pdf?seq uence=1>. Acesso em: 29 jun. 2016.

CAMPOS, J.; SANTACHÈ, A.; TEIXEIRA, C. Visualização de modelos tridimensionais de sistemas de informações geográficas distribuídos baseados na WEB. In: BRAZILIAN WORKSHOP ON GEOINFORMATICS, 1999, Campinas. Proceedings... São José dos Campos: INPE, 1999. p. 50-58.

GRUBER, T. R. A translation approach to portable ontology specifications. Technical Report KSL92-71. Stanford: Knowledge Systems Laboratory. Stanford University, 1993. Disponível em: <http://wwwksl.stanford.edu/KSL_Abstracts/KSL- 92-71.html>

GUARINO, N. Understanding, building and using ontologies. In: PROCEEDINGS OF KNOWLEDGE ACQUISITION FOR KNOWLEDGEBASED SYSTEMS WORKSHOP. 10. 1996.

GUARINO, N. Formal ontology and information systems. In: INTERNATIONAL CONFERENCE ON FORMAL ONTOLOGY IN INFORMATION SYSTEMS FOIS'98, 1998, Trento. Proceedings... Amsterdam: IOS Press, 1998. p. 3-15.

HEATH, T.; BIZER, C. Linked data: evolving the web into a global data space. Morgan \& Clarepool, 2011.

HORROCKS, I. et al. SWRL: A Semantic Web Rule Language Combining OWL and RuleML. 2004. Disponível em: <https://www.w3.org/Submission/SWRL/>. Acesso em: 20 jun. 2016. 
SANTAREM SEGUNDO, J. E. Representação Iterativa: um modelo para repositórios digitais. 2010. 224 f. Tese (Doutorado em Ciência da Informação) - Faculdade de Filosofia e Ciências, Universidade Estadual Paulista, Marília. 2010.

SANTAREM SEGUNDO, J. E. Web Semântica: introdução a recuperação de dados usando SPARQL. In: ENCONTRO NACIONAL DE PESQUISA EM CIÊNCIA DA INFORMAÇÃO: além das nuvens, expandindo as fronteiras da Ciência da Informação, 15., 2014. Belo Horizonte. Anais... Belo Horizonte: UFMG/ ECI, 2014. p. 3863-3882.

SANTAREM SEGUNDO, J. E.; CONEGLIAN, C. S. Tecnologias da Web Semântica aplicadas a organização do conhecimento: padrão SKOS para construção e uso de vocabulários controlados descentralizados. In: Organização do Conhecimento e Diversidade Cultural. Marília: Fundepe, 2015, v. 3, p. 224-233. Disponível em: <http://isko-brasil.org.br/wpcontent/uploads/2015/09/Organiza\%C3\%A7\%C3\%A3o-do-Conhecimento-eDiversidade-Cultural-ISKO-BRASIL-2015.pdf>. Acesso em: 25 jun. 2016.

SANTAREM SEGUNDO, J. E. Web semântica, dados ligados e dados abertos: uma visão dos desafios do Brasil frente às iniciativas internacionais. Tendências da Pesquisa Brasileira em Ciência da Informação, v. 8, n. 2, 2015. $<$ http://inseer.ibict.br/ancib/index.php/tpbci/article/view/207>. Acesso em: 25 jun. 2016.

SHADBOLT, N.; BERNERS-LEE, T.; HALL, W. "The Semantic Web Revisited," in IEEE Intelligent Systems, v. 21, n. 3, p. 96-101, Jan.-Feb. 2006.

SPARQL. Sparql 1.1 Overview. W3C, 2013. Disponível em <http://www.w3.org/TR/2013/REC-sparql11-overview-20130321/>. Acesso em: 13 jun. 2016.

\section{Title}

Semantic web and ontologies: a study on construction of axioms and use of inferences

\section{Abstract:}

Introduction: The Semantic Web provides technologies and concepts that go through by issues such as description, representation and queries of data. In this scenario, ontologies contextualize the information, allowing generation of inferences. The OWL language has features that facilitate the inclusion of logic in the data, it is an essential element for the Semantic Web and Linked Data. However, the question is: how these inferences can be viewed and used in the Semantic Web.

Objective: To analyze the inference types in the context of Semantic Web technologies, and consequently to the Linked Data. 
Methodology: It consisted in two parts, the first a literature review for discussion of the topic, and the second an exploratory study based on concepts proof and inferences testing.

Results: We identify and present four embodiments of inferences, making tests to prove their application.

Conclusions: The use of inferences in ontologies can occur in many ways and there is a variation in the expression that the types of inferences have. We found that inferences can add logic to the representations of the data, since the contextualization of information using ontologies contains axioms, increasing the representational capacity of information when using inferences. Noteworthy is the OWL language as a reference for enrichment and integration of axioms in the construction of ontologies.

Keywords: Semantic Web. Linked data. Inferences. Ontologies. Sparql.

\section{Titulo}

Web semántica y ontologías: un estudio sobre la construcción de axiomas y el uso de inferencias

\section{Resumen:}

Introducción: La Web Semántica proporciona tecnologías y conceptos que abarcan cuestiones como la descripción, representación y consulta de datos. En este escenario, las ontologías contextualizan las informaciones, permitiendo la generación de inferencias. El lenguaje OWL presenta características que facilitan la inserción de lógica en los datos, siendo este lenguaje un elemento esencial para la Web Semántica y Linked Data. Sin embargo, la pregunta que surge es: cómo estas inferencias pueden ser visualizadas y utilizadas en la Web Semántica.

Objetivo: Analizar los tipos de inferencia en el contexto de la Web Semántica, y en consecuencia de Linked Data.

Metodología: Fue divida en dos partes, siendo la primera un levantamiento bibliográfico para la discusión del tema, y en la segunda un estudio exploratorio basado en pruebas de conceptos y tests de uso de inferencias.

Resultados: Se identificaron y presentaron cuatro formas de realización de inferencias, realizando pruebas que demuestran su aplicación.

Conclusiones: El uso de inferencias en ontologías puede ocurrir de múltiples formas existiendo una variación en el formato que los tipos de inferencias poseen. Encontramos que las inferencias pueden añadir lógica en las representaciones de los datos, ya que la contextualización de las informaciones con el uso de ontologías contiene axiomas, aumentándose la capacidad de representación con el uso de inferencias. Se destaca el lenguaje OWL como referencia para el enriquecimiento e inserción de axiomas en la construcción de ontologías.

Palabras-clave: Web Semântica. Linked Data. Inferências. Ontologias. Sparql.

Enviado em: 17.07.2016.

Aceito em: 20.11.2016.

Inf. Inf., Londrina, v. 21, n. 2, p. 217 - 244, maio/ago., 2016. 